\title{
Tropical sprue in a case of heterosexually transmitted acquired immune deficiency syndrome
}

\author{
Raymond, G. LAhaie, MD, FRCPC, Sidney SABbah, MD, FRCPC, ANDRÉ Dumont, MD, CSPQ \\ Pierre Poitras, MD, FrCPC, Michel Gagnon, MD, FrCPC, François Martin, MD, FrCPC, \\ MiCHEL BOIVIN, MD, FRCPC, MiCHEL LEMOYNE, MD, CSPQ
}

ABSTRACT: A heterosexual patient presented with severe diarrhea, malabsorption and weight loss. He was found to have acquired immune deficiency syndrome (AIDS) on the basis of candida esophagitis, Pneumocystis carinii pneumonia, inversion of T lymphocyte subpopulation ratio and the presence of serum anti-HIV virus antibodies. The patient had travelled to Haiti and the Dominican Republic where he had multiple sexual contacts with indigenous women. Despite thorough evaluation to identify the cause of diarrhea no infectious agent was discovered. Duodenal biopsy showed partial villous atrophy, slight crypt hyperplasia and increased number of intraepithelial lymphocytes. After two weeks treatment with tetracycline and folic acid, the patient was symptom free and intestinal histology had returned to normal. Tropical sprue must thus be considered in the differential diagnosis of intestinal villous atrophy in patients with AIDS. Can J Gastroenterol 1988;2(2):67-70

Key Words: AIDS, Tropical sprue, Villous atrophy

S INCE THE RECENT RECOGNITION OF acquired immune deficiency syndrome (AIDS), there have been many reports of infectious, inflammatory and tumoral involvement of the gastrointestinal tract in association with this disease (1-4). Indeed, diarrhea and weight loss are common complaints among AIDS patients and can at times be the predominant clinical problem. While in certain of these subjects specific infectious agents can be found $(2,3)$, in others, no infec- tious etiology can be ascertained despite extensive clinical and microbiological investigation. In the latter group, jejunal and colorectal abnormalities have been reported $(5,6)$. Of the jejunal abnormalities described, one has been termed pseudo-Whipple's disease in that mucosal biopsies demonstrate abundant foamy macrophages. This entity is thought to result from gastrointestinal involvement in a systemic Mycobacterium avium infection (7).
Department of Medicine (Gastroenterology) and Pathology. Saint-Luc Hospital, Montreal, Quebec Correspondence and reprints: Dr Raymond G. Lahaie, André-Viallet Clinical Research Centre, 1058 St Denis Street, Montreal, Quebec H2X 3/4. Telephone (514) 281-2470

Received for publication February 12, 1988. Accepted February 26, 1988
A second type of jejunal involvement, in this case of unknown etiology, results in a partial villous atrophy associated with crypt hyperplasia and increased number of intraepithelial lymphocytes (5). Although this histology could suggest the diagnosis of sprue, neither a gluten-free diet nor antibiotic treatment has resulted in clinical or histological remission of the jejunal anomaly or of the consequent diarrhea and malabsorption $(5,8)$.

A case of a patient with documented AIDS, who presented such a histological jejunal abnormality with an associated malabsorption is reported. Because he had been travelling to an area where tropical sprue is known to be endemic and as there is no known treatment for AIDS-related enteropathy, a treatment known to give good results in tropical sprue was attempted.

\section{CASE PRESENTATION}

A 41-year-old, white, French Canadian, exclusively heterosexual male, was in good health, except for moderate obesity $(95 \mathrm{~kg}$ ) until March 1985 when he developed nonbloody diarrhea with a stool frequency of eight to 12 bowel movements per day. At that time, he had recently returned from a two-month vacation to the Dominican Republic and 
Haiti where he had been travelling on regular occasions for the past five years. While abroad he had had multiple sexual contacts with indigenous women. He was asymptomatic during and after previous trips only to develop diarrhea within two weeks of his last return.

When the patient first presented at the emergency ward he was dehydrated, had lost 4 to $5 \mathrm{~kg}$ and had slight hypokalemic acidosis. At that time, stool cultures revealed the presence of Campylobacter fetus subspecies jejuni. The patient was treated with erythromycin with a transitory clinical response.

He subsequently returned to the emergency ward in April 1985 complaining of the same symptoms. Stool cultures for bacteria, including Clostridium difficile were negative whereas stool examinations for ova and parasites revealed the presence of Giardia lamblia. Small bowel series showed a moderate dilatation of the proximal small bowel. The patient was treated with metronidazole and once again the response was transitory, the diarrhea recurring two weeks later.

Despite recurrence of the diarrhea, the patient consulted again only in July 1985 . At this time he complained of fever, nonproductive cough, dyspnea and anorexia. He still had six to eight loose, watery, nonbloody stools per day and presented a weight loss of $30 \mathrm{~kg}$. On physical examination the patient appeared chronically ill; he was febrile $\left(38^{\circ} \mathrm{C}\right)$. There were no cervical or axillar adenopathies. Heart and lung examination was normal as was the abdominal examination. Rectal exam including rectosigmoidoscopy was normal except for a superficial acute anal fissure.

Routine laboratory investigations revealed a normal blood hemoglobin at $14.4 \mathrm{~g} / \mathrm{dL}$, a peripheral leukocyte count at $6700 / \mathrm{mm}^{3}$ with $78 \%$ neutrophils, $16 \%$ lymphocytes and a normal platelet count. No hypersegmented neutrophils were seen on peripheral smears. Blood urea nitrogen, creatinine, glucose level and liver function tests as well as urinalysis were normal. Protein electrophoresis showed only slight hypoproteinemia and hypoalbuminemia while quantitative immunoglobulin levels were within normal limits. Coagulation tests were normal. Chest $\mathrm{x}$-ray showed a minimal bibasilar reticular infiltrate. Stool cultures and search for ova and parasites, including Cryptosporidium species, were negative.

With this data at hand, it was decided to obtain directly a small bowel mucosal biopsy through the gastroduodenoscope. Upper gastrointestinal endoscopy revealed multiple white, spotty exudates lining the entire esophageal wall, brushings and biopsies of which showed the presence of Candida albicans and an associated esophagitis. Concurrent distal duodenal mucosal biopsies revealed subtotal villous atrophy with slight crypt hyperplasia and increased intraepithelial lymphocytes (Figure 1). No Giardia or Cryptosporidia were seen on the epithelial surface. Rectal biopsy was normal. Subsequent complementary investigations revealed a blood carotenemia at 6 $\mathrm{mg} / 100 \mathrm{~mL}$, folic acid at $1.11 \mathrm{mg} / 100 \mathrm{~mL}$ (normal 1.7 to 15.5 ) with a normal $\mathrm{B}_{12}$ and $\mathrm{a} 2 \mathrm{~h}$ blood D-xylose of $6 \mathrm{mg} / 100 \mathrm{~mL}$ (normal, more than 25). Transbronchial lung biopsy showed numerous Pneumocystis carinii. Serology gave a negative VDRL and HBsAg antigen whereas antibodies to HIV virus were positive. T helper lymphocytes were 392 cells/ $\mathrm{mm}^{3}$, T suppressor lymphocytes 854 cells $/ \mathrm{mm}^{3}$ and $T$ helper to $T$ suppressor ratio was depressed at 0.45 .

The patient was treated with intravenous trimethoprim-sulfamethoxasole for the Pneumocystis carinii penumonia and with ketoconazole for the candida esophagitis. He responded well to both treatments. As for the diarrhea, with subtotal duodenal atrophy, it was hypothesized that he may have acquired tropical sprue during his travels to a known endemic area and was therefore treated with the combination of tetracycline and folic acid. He responded very well to this therapy and became asymptomatic within 10 days. Subsequent distal duodenal mucosal biopsy repeated 20 days after the beginning of the treatment showed an almost complete reversal of the duodenal villous atrophy (Figure 2 ).

The patient was subsequently dis-
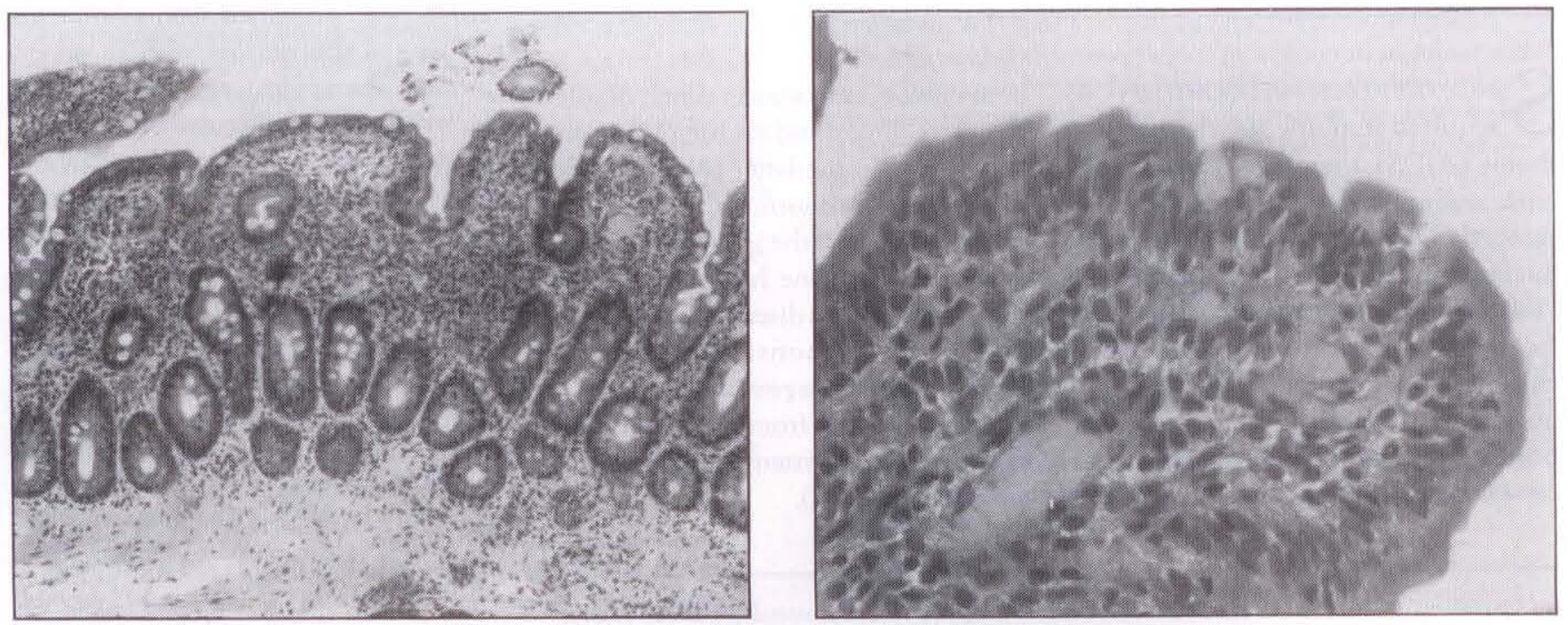

Figure 1) Endoscopic duodenal biopsy taken at original presentation. Left Subtotal villous atrophy with broadening of villi and infiltrations with chronic inflammatory cells (HPS $\times$ 70). Right Surface epithelium with low columnar and cuboidal epithelial cells with the associated inflammatory infiltrate (HPS $\times 300$ ) 

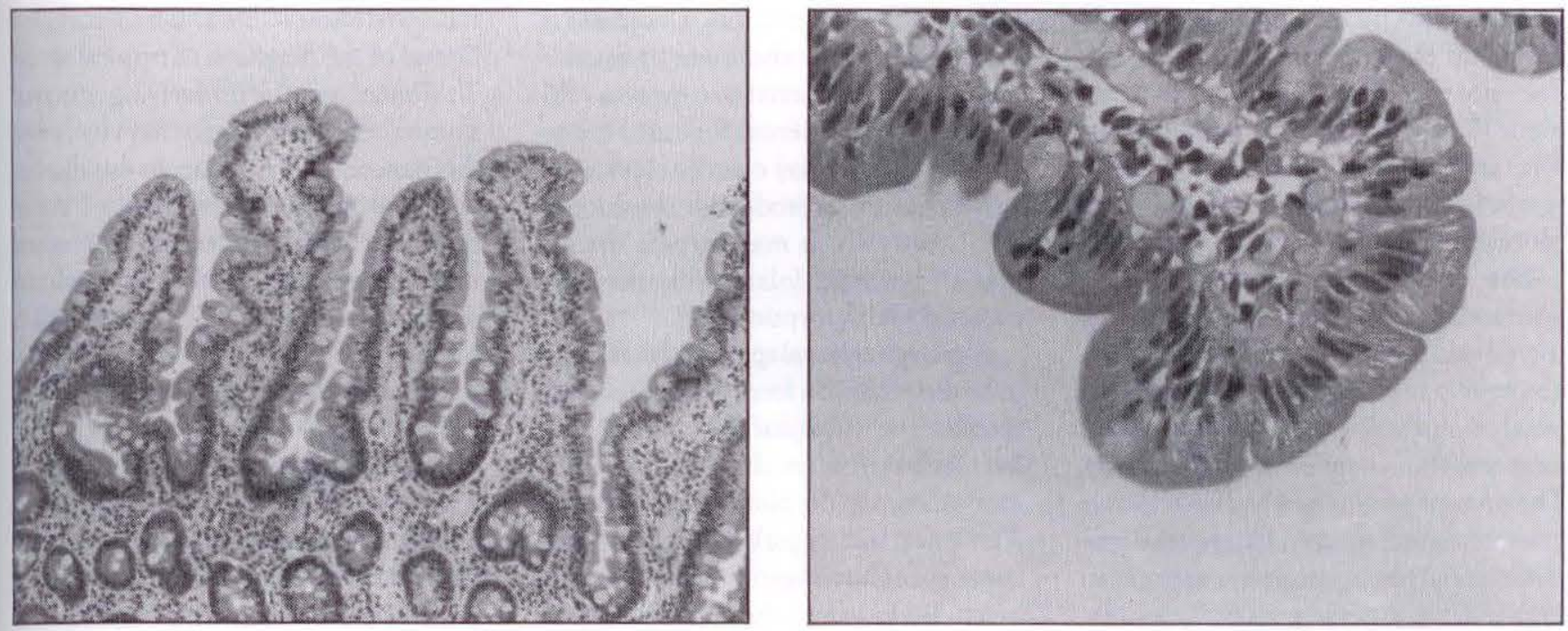

Figure 2) Endoscopic duodenal biopsies obtained after clinical remission showing (left) almost complete restitutions of villous architecture (HPS $\times 70$ ) and (right) regression of surface epithelium abnormalities (HPS $\times 300$ )

charged from hospital and followed as an outpatient. Six months later, he had regained the $30 \mathrm{~kg}$ which he had lost during his illness and remained perfectly healthy taking no medication. At this time, his T helper to T suppressor ratio remained depressed at 0.43 and he remained anergic to common antigens. At endoscopy, to obtain a control intestinal biopsy, he was found to have several small white plaques in the esophagus which were shown to be Candida albicans on cytological smears. Intestinal biopsy taken at the same endoscopic session showed normal duodenal histology (Figure 2). D-xylose absorption had also returned to normal. Although asymptomatic he was given a two week course of oral nystatin. Two months later he remained well and without symptoms.

Fifteen months after his original presentation he developed cyclic fever followed by headaches of progressive intensity. He had no gastrointestinal symptoms. Appropriate investigations revealed cerebral toxoplasmosis for which he was treated with pyrimethamine and sulfamethoxasole. He succumbed to this opportunistic infection two months later without recurrence of the gastrointestinal problems.

\section{DISCUSSION}

The association of an acquired defect in cellular immunity, demonstrated by an absolute lymphopenia ( 1070 lympho- cytes $/ \mathrm{mm}^{3}$ ) selective for $\mathrm{OKT}_{4}$ helper/ inducer lymphocytes, with the documentation of three opportunistic infections including Pneumocystis carinii pneumonia, Candida albicans esophagitis and cerebral toxoplasmosis, meets the Centre for Disease Control criteria for the diagnosis of AIDS. The diagnosis of AIDS in this case was further supported by the presence of a definite risk factor in that the patient had multiple heterosexual relations with Haitians in whom the prevalence of HIV infections seems inordinately elevated $(9,10)$. Furthermore the presence of HIV antibodies confirms previous contact with human $T$ cell lymphotropic retrovirus thought to be the infectious agent responsible for this disease (11). A second important criteria in the Centre for Disease Control's definition of AIDS specifies that no other identifiable cause can be found for the underlying immunodeficiency (12).

In the present case, the patient presented with severe intestinal malabsorption with the resulting protein-calorie malnutrition which produced a loss of $30 \%$ of his original body weight. Such protein-calorie malnutrition is known to induce immunodeficiency states. Indeed, decreased total T lymphocyte counts, T helper and suppressor counts and $\lg \mathrm{A}$ secretion have been described in such states of malnutrition (13). Protein-calorie malnutrition has also been thought to be an important factor in epidemics of
Pneumocystis carinii pneumonia in malnourished children (14). In this case, the role of the malabsorption, with its consequent immunodeficiency, on the opportunistic infections sustained by the patient can only be speculated. Superimposition of severe malabsorption may have further depressed his immune system and rendered him more susceptible to opportunistic infections.

The duodenal villous atrophy with concurrent nutrient malabsorption observed is by no means specific and diagnostic of tropical sprue. Several infectious and noninfectious enteropathies could very well have given the same clinical and histological picture. Of the intestinal infections known to produce similar villous patterns, both giardiasis and cryptosporidiosis were excluded by the study of multiple stool specimens and endoscopic duodenal aspirates looking specifically for these pathogens. Furthermore, intestinal biopsy showed no evidence of parasites adhering to the enterocytes. Systemic Mycobacterium avium infection can be excluded on the basis of histology as this infection has been shown to produce a histological picture resembling Whipple's disease with an accumulation of histiocytes in the lamina propria. The difference from Whipple's disease, however, is that these histiocytes contain numerous acid-fast rods (7). The AIDS-related enteropathy, for which there is yet no etiology and which gives 
a histological picture undifferentiable from that observed in this patient (5), does not respond to tetracycline treatment $(5,8)$. However, tetracycline and folic acid therapy resulted in a rapid resolution of the jejunal inflammatory process in the case described here.

The response observed following antibiotic treatment could also suggest the possibility of the syndrome of malabsorption resulting from intestinal bacterial overgrowth associated with a blind loop or an intestinal motor abnormality. The present patient had had no previous gastrointestinal surgery, had normal gastrointestinal roentgenograms, apart from slight dilatation of the proximal jejunum, and had no systemic disease known to alter intestinal motility which mitigates against this possibility. Furthermore, although decreases in intestinal secretory IgA have been shown to occur in AIDS patients (15), bacterial overgrowth has yet to be reported as a cause of malab-

\section{REFERENCES}

1. Gottlieb MS, Groopman JE, Weinstein WM, Fahey JL, Detels R. The acquired immunodeficiency syndrome. Ann Intern Med 1983:99:208-20.

2. Baker RW. Pepperiorn MA. Gastrointestinal ailments of homosexual men. Medicine 1982;61:390-405.

3. Bories C, Salmeron M, Modigliani R. Diarrhée et syndrome d'immunodépression acquise. Gastroenterol Clin Biol 1985;9:354-60.

4. Réné E, Marche C, Regnier B, et al. Manifestations digestives du syndrome d'immunodéficience acquise (SIDA): Étude chez 26 patients. Gastroenterol Clin Biol 1985;9:327-35.

5. Kotler DP, Gaetz HP, Lange M, Klein EB, Holt PR. Enteropathy associated with the acquired immunodeficiency syndrome. Ann Intern Med 1984;101:421-8.

6. Gillin JS, Shike M, Alcock N, et al. Malabsorbtion and mucosal abnormalities of the small intestine in the acquired sorption in these patients. Decreases in serum folic acid is also quite unusual in such cases of bacterial overgrowth (16). On the contrary, serum folic acid is usually normal and may even be elevated as a result of a folate production, by the bacterial flora, which may surpass the degree of bacterial folate utilization and mucosal malabsorption (17).

Although tropical sprue is also thought to be the result of a form of bacterial overgrowth, the intestinal flora responsible for this lesion seem different from that encountered in the blind loop syndrome. The bacterial population in tropical sprue is not heterogeneous as is the case in a blind loop and the clinical and biochemical manifestations of the two entities differ.

The association of travel to an endemic area, a compatible histological picture and associated malabsorption pattern with the rapid reversal of the pathological process after the therapeutic combi-

immunodeficiency syndrome. Ann Intern Med 1985;102:619-22.

7. Gillin JS, Urmacher C, West R, Shike M. Disseminated Mycobacterium avium-intracellular infection in acquired immunodeficiency syndrome mimicking Whipple's disease. Gastroenterology 1983;85:1187-91.

8. Masur $H$. The acquired immunodeficiency syndrome. DM 1983;30:1-48.

9. Vieira J, Frank E, Spira TJ, Landesman $\mathrm{SH}$. Acquired immune deficiency in Haitians: Opportunistic infections in previously healthy Haitian immigrants. N Engl J Med 1983;308:125-9

10. Pitchenik AE, Fische MA, Dickinson GM. Opportunistic injections and Kaposi's sarcoma among Haitians: Evidence of a new acquired immunodeficiency state. Ann Intern Med 1983;98:277-84.

11. Gallo RC, Wong-Staal F. A human T-lymphotropic retrovirus (HTLV-111) as the cause of the acquired immunodeficiency syndrome. Ann nation of tetracycline and folic acid, is in favour of the diagnosis of tropical sprue. To what extent the underlying acquired immunodeficiency might have increased this patient's susceptibility to this disease, which probably results from a form of intestinal bacterial overgrowth, remains unanswered. The study of the prevalence of tropical sprue in Haitians with AIDS or AIDS-related complex could possibly serve to answer this question.

This case of tropical sprue in a patient with AIDS has important therapeutic implications. Although therapy with tetracycline is generally ineffective in the treatment of AIDS-related enteropathy, patients presenting with this clinical and histological picture who report a history of recent travel to areas of the world where tropical sprue is endemic may benefit from such a treatment. It may be more important to consider this possibility in Haitians with AIDS and jejunal villous atrophy.

Intern Med 1985; 103:679-89

12. Fauci AS, Macher AM, Lango DL, et al. Acquired immunodeficiency syndrome: Epidemiology, clinical immunologic and therapeutic considerations. Ann Intern Med 1984;100:92-106.

13. Chandra RK. Nutrition, immunity and infection: present knowledge and future directions. Lancet 1983;i:688-91.

14. Hughes WT, Price RA, Sisko F, et al. Protein caloric malnutrition: A host determinant for Pneumocystic carinii infection. Am J Dis Child 1974;128:44-52

15. Kotler DP, Scholes JV. Altered intestinal plasma cell immunoglobulins in the acquired immunodeficiency syndrome. Gastroenterology 1984:86:1144.

16. Berk JE, Hanbrich WS, Ralser MH, Roth JLA, Schaffner F. Bockus Gastroenterology, 4th edn. Philadelphia: WB Saunders Co, 1985.

17. Hoffbrand AV, Tabaqchali S, Booth CC, Molin DL. Small intestinal bacterial flora and folate status in gastrointestinal disease. Gut 1971;12:27-33. 


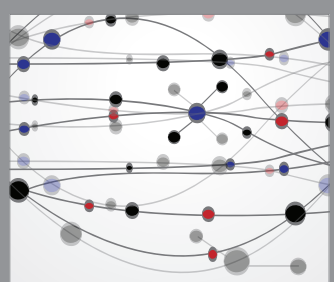

The Scientific World Journal
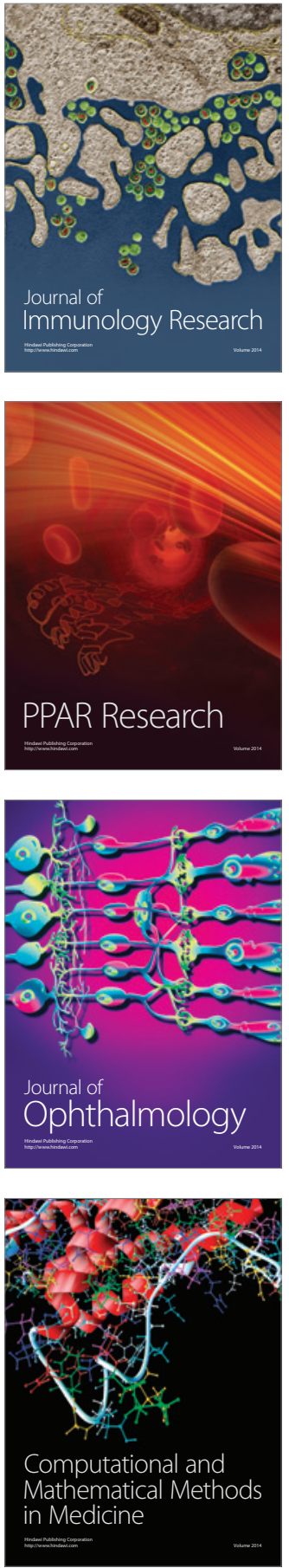

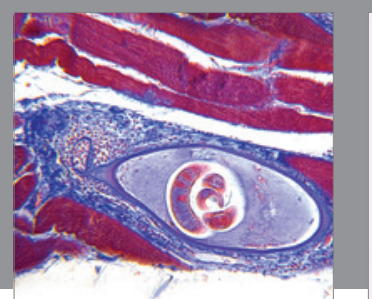

Gastroenterology Research and Practice

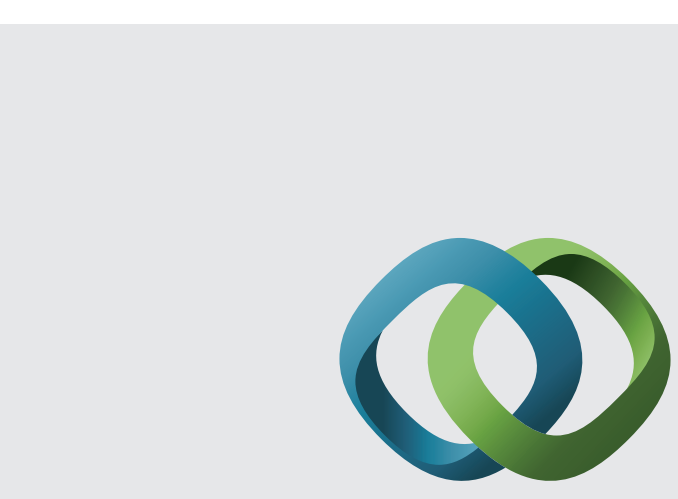

\section{Hindawi}

Submit your manuscripts at

http://www.hindawi.com
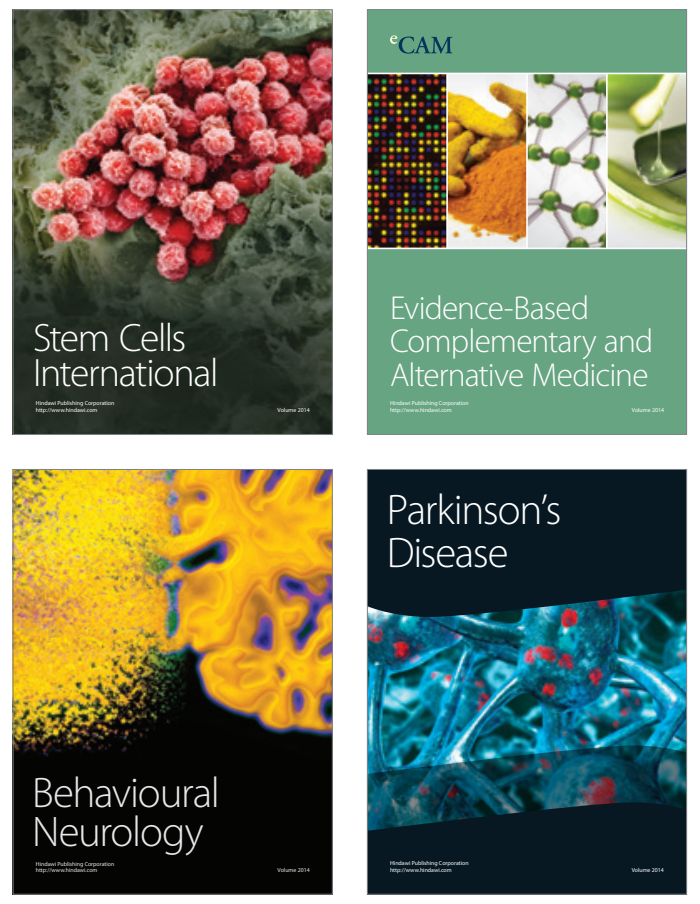
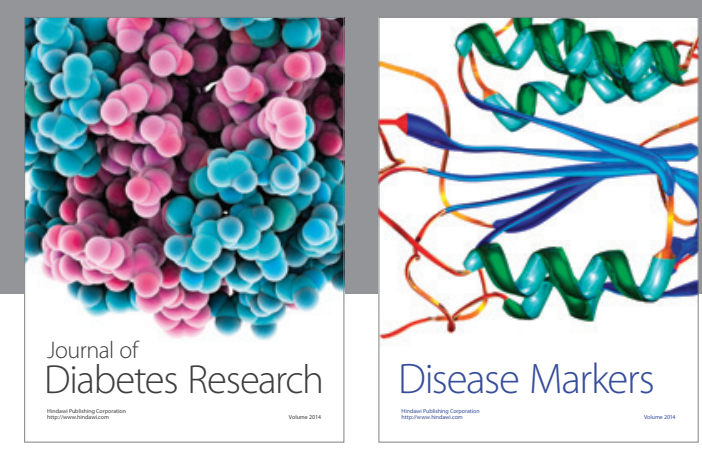

Disease Markers
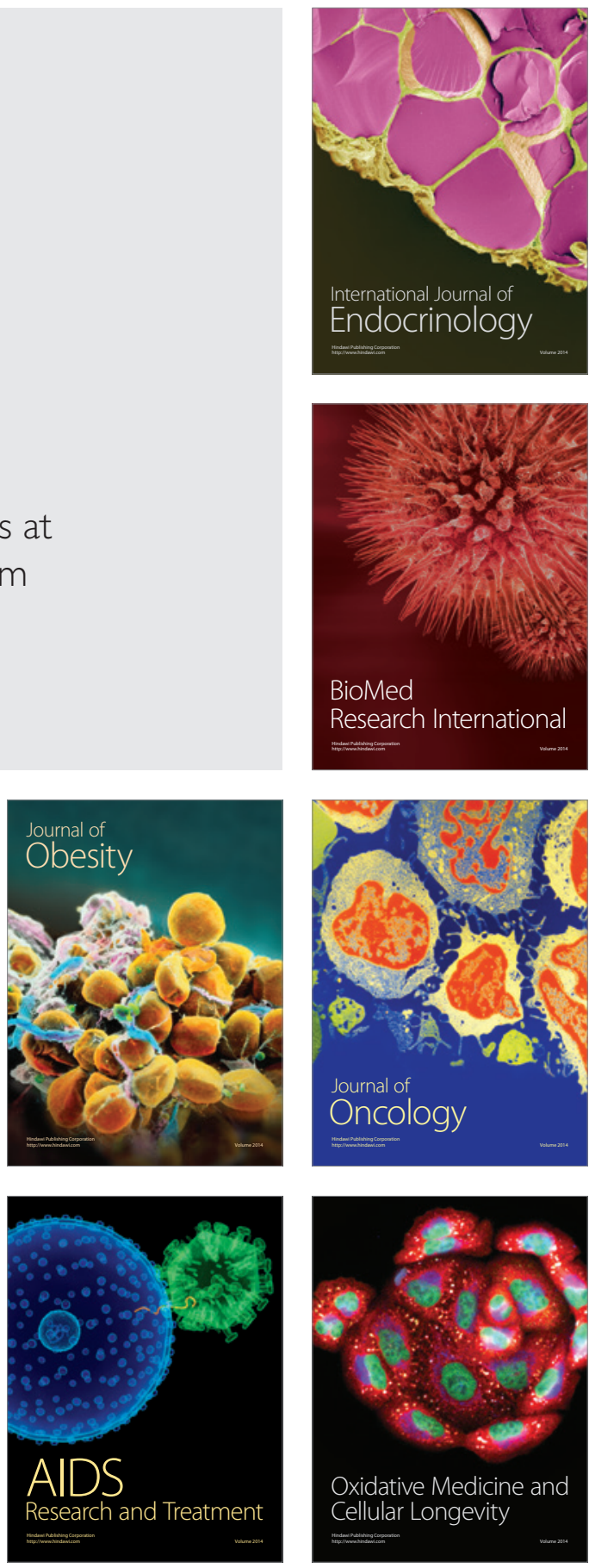